\title{
Retinal thickness change after focal laser treatment of diabetic macular oedema
}

Department of Ophthalmology and Visual Sciences, UIC Eye Center, University of Illinois at Chicago, Chicago, Illinois, USA M Shahidi N P Blair

Department of Ophthalmology, Kyoto University Faculty of Medicine, Kyoto 606, Japan

Y Ogura

Department of Ophthalmology, Wilmer Ophthalmological Institute, Johns Hopkins University, Baltimore, Maryland, USA R Zeimer

Correspondence to: Mahnaz Shahidi, $\mathrm{PhD}$ Department of Ophthalmology and Visual Sciences, University of Illinois at Chicago, 1855 West Taylor Street, Chicago, Il 60612, USA.

Accepted for publication 7 July 1994

\begin{abstract}
Laser photocoagulation has been used successfully for the treatment of clinically significant macular oedema to reduce the risk of loss of vision in diabetic patients. A quantitative method for measuring retinal thickness was applied to 20 patients with diabetic macular oedema before and 4 months after focal laser treatment to assess the reduction in retinal thickening and its relation to visual acuity. The degree of thickening at each location, defined by thickness index, was determined relative to the corresponding average value in normal subjects. Comparison of quantitative retinal thickness measurements before and after treatment demonstrated that treatment at thickness indices of approximately $1.6(60 \%$ thickening) has nearly $50 \%$ probability for reversal of thickening to within the normal range $(\leqslant 1 \cdot 3)$, whereas at thickness indices greater than $2.8(180 \%$ thickening) there is less than $2 \cdot 5 \%$ probability that reversal will occur. The level of foveal thickening before treatment strongly correlated with the degree of thickening after treatment. Most of the eyes with an improvement in visual acuity had a foveal thickness within the normal range at 4 months' follow up. These findings suggest that quantitative retinal thickness measurement provides an objective assessment of the degree of macular oedema and can be useful for monitoring the efficacy of focal laser treatment in reducing the thickening and relating the latter to visual outcome.
\end{abstract}

(Brf Ophthalmol 1994; 78: 827-830)

Macular oedema is considered the leading cause of decreased vision in patients with diabetic retinopathy.$^{1-3}$ It is caused by the breakdown of the blood-retinal barrier which permits the leakage of fluid and lipoproteins (exudates) into the retina, causing retinal fluid accumulation and thickening. Clinical indications of macular oedema include macular thickening, presence of exudates, and leakage determined by fluorescein angiography. Numerous studies have supported the beneficial effect of photocoagulation in reducing the rate of loss of vision in eyes with macular oedema. ${ }^{48}$ The Early Treatment Diabetic Retinopathy Study (ETDRS) demonstrated conclusively that focal laser treatment of macular oedema, as diagnosed by clinical evaluation of retinal thickening, effectively reduces the risk of loss of vision and decreases the frequency of persistent macular oedema. ${ }^{9}$ Classification of diabetic retinopathy has been reported according to the presence of microaneurysms/exudates/ haemorrhages on fundus photographs and leakage on fluorescein angiograms. ${ }^{10} 11$ Owing to the lack of a quantitative method for assessment of macular thickening, the degree and change in retinal thickening in response to treatment have not been established. We developed a method for retinal thickness measurement and applied it to quantitate thickening caused by macular oedema in diabetic patients. ${ }^{12}$ In the present study, we applied the same method to diabetic patients with macular oedema before and after focal laser treatment to determine the change in retinal thickness and its relation to improvement in visual acuity at 4 months' follow up.

\section{Materials and methods}

Twenty patients with diabetes type I or II, who were diagnosed as having clinically significant diabetic macular oedema in one eye as defined by ETDRS criteria ${ }^{9}$ were recruited. All patients had focal maculopathy and, in addition, some patients also had diffuse leakage. The patients had visual acuities of $20 / 80$ or better, clear media, and absence of neovascularisation and had not undergone previous panretinal photocoagulation treatment. The patients' ages ranged from 47 to 72 years (mean 62 (SD 7) years). Following routine clinical fluorescein angiography, focal laser photocoagulation was applied according to the ETDRS treatment guidelines. Focal and grid laser treatment was applied to focal leakage sites and diffuse areas of leakage and non-perfusion, respectively. The patients returned for follow up after 4 months.

Before the initiation of the study, an approval from the institutional review board (IRB) for use of human subjects in the study was obtained. The study was explained to each patient, and signed consent forms were obtained. Before the retinal thickness measurements were performed, the eye was dilated to a minimum of $6 \mathrm{~mm}$ with drops of $2.5 \%$ phenylephrine hydrochloride and $1 \%$ tropicamide. The cornea was anaesthetised with one drop of proxymetacaine (proparacaine) hydrochloride.

The retinal thickness analyser was used quantitatively to measure retinal thickness. ${ }^{13}$ The instrument consisted of a green $\mathrm{HeNe}$ laser, operating at a wavelength of $543 \mathrm{~nm}$, mounted on a slit-lamp biomicroscope. The expanded laser beam was focused on the retina by a combination of the biomicroscope objective and a cylindrical lens, thereby creating a slit. An image of the intersection of the laser slit with the anterior and posterior surfaces of the retina was recorded on film and later digitised. The separation between the two intersections was determined with a customised software program developed by one of us (MS). The separation was converted to thickness units with use of an empirically derived calibration factor. 
Retinal thickness measurements were performed on average at five locations in each eye. The measured locations were half a disc diameter in size and within one disc diameter of the centre of the fovea. The sites of the measurements were predetermined by using a fixation target presented to the subject during the measurement and regardless of the degree of thickening. Following the measurement, the exact locations of the thickness measurements were recorded on an enlarged red free fundus photograph. Retinal thickness was measured before focal laser treatment and 4 months after treatment.

A retinal thickness index was defined at each location by normalising the value to the corresponding average thickness value found in an agematched control population, consisting of 16 normal subjects with an age range of 40 to 60 years. A foveal thickness index was defined as the averaged measurements on three slits located within 0.5 disc diameter of the fovea, divided by the corresponding value in normal subjects. Retinal thickness was considered to be abnormal when the value was outside the $97.5 \%$ confidence interval for normal thickness, which corresponds to a retinal thickness index greater than $1 \cdot 3$. The variability of thickness measurements acquired by this method was assessed in normal subjects and found to be $8 \% .^{14}$ Thickness measurements obtained at the same location before and after treatment were compared, as a first approximation, by performing linear regression of the data points to determine the correlation coefficient indices. The slope and intercept were calculated and used to plot the least squares fit line. The $95 \%$ prediction confidence interval for the foveal thickness index after treatment was calculated. ${ }^{15}$ Visual acuity was measured in each eye according to ETDRS protocol by an observer masked to the retinal thickness data. Refraction was performed with a trial frame and a modified Bailey-Lovie chart in a room with 75 foot candle illumination. The best corrected visual acuity was recorded and a change of one line or more was considered significant. The association between foveal thickness and visual acuity was examined with Fisher's exact test to determine the level of significance.

indices greater than $2 \cdot 8$ had

less than $2.5 \%$ probability

for reversing the thickening to

normal (thickness index

$\leqslant 1 \cdot 3$ ) following treatment.

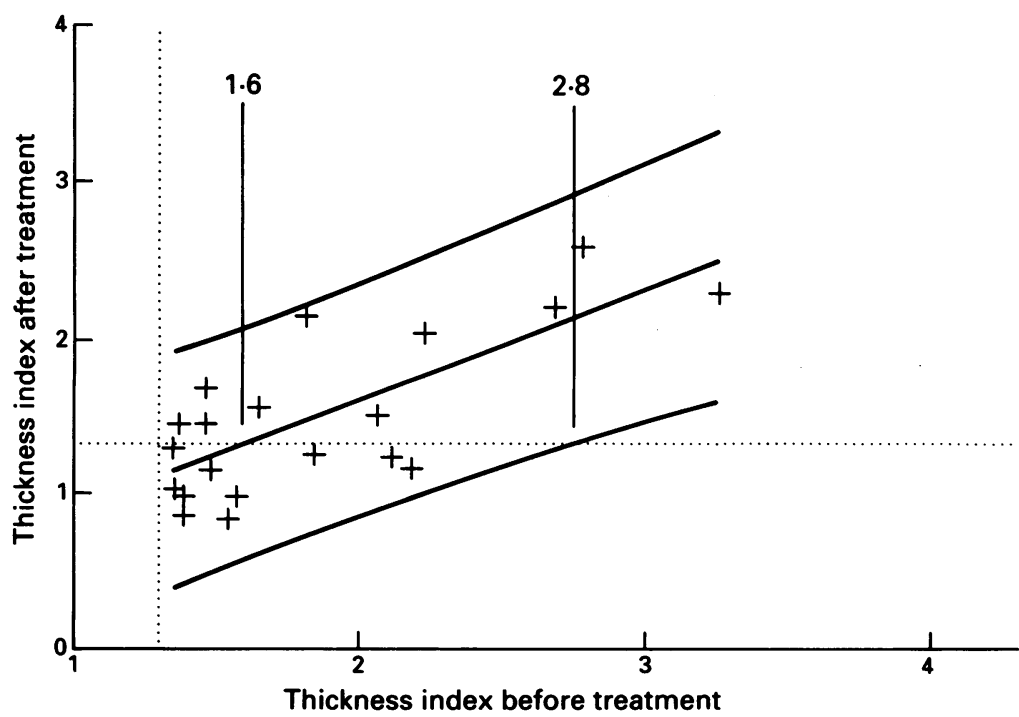

Results

Nineteen (86\%) of 22 thickened locations before treatment showed improvement or stabilisation following treatment, and thickening was resolved in 11 locations. The pretreatment thickness index for locations that returned to normal was $1.62(0.30)(n=11)$. Three $(3 \%)$ of 89 locations with a pretreatment macular thickness index within the $95 \%$ confidence interval for normal thickness $(\leqslant 1 \cdot 3)$ developed macular thickening outside the normal range $(>1 \cdot 3) 4$ months after treatment. The thickness measurements obtained in the region outside 0.5 disc diameter from the centre of the fovea 4 months after treatment were plotted as a function of the thickness before treatment for the thickened locations (Fig 1). From the data, the thickness index after treatment was predicted and the $95 \%$ prediction confidence limits of the interval have been plotted as the broken lines (Fig 1). For example, a pretreatment thickness index of 1.6 will result in a thickness index within the prediction confidence interval following treatment, with the centre of the interval corresponding to a thickness index of $1 \cdot 3$, depicted by the horizontal dotted line (Fig 1). On the other hand, for a pretreatment thickness index of $2 \cdot 8$, the lower limit of the interval coincides with a thickness index of 1.3 following treatment. Thus, treatment of maculas with pretreatment thickness indices of 1.6 had approximately a $50 \%$ probability of reversing the thickness to within the normal range (thickness index $\leqslant 1 \cdot 3$ ), while a macula with a thickness index greater than $2 \cdot 80$ had less than a $2.5 \%$ probability of returning to normal with one treatment.

Foveal thickness was decreased in all eyes with above normal thickness. The pretreatment foveal thickness index for the eyes in which the thickness returned to normal was $1.67(0.31)$ $(n=4)$. In all 12 eyes $(100 \%)$ with a pretreatment thickness within the normal range, it remained within the normal range for 4 months following treatment. The thickness measurements obtained at the fovea before and 4 months after treatment were plotted (Fig 2). Linear regression of the data points yielded a correlation coefficient: $r=0.89(\mathrm{p}<0.01)$. From the prediction confidence interval, the probability of reversing the foveal thickness index of 1.6 to within the normal range was $50 \%$.

Foveal thickness and visual acuity were measured before and after treatment in all eyes (Table 1). The visual acuity improved by one line or more in eight $(67 \%)$ of 12 eyes with pretreatment visual acuities of 20/25 or worse, and seven of these eight eyes had a foveal thickness index within the normal range at the 4 month follow up visit. The degree of foveal thickening before treatment was compared with the visual acuity outcome at 4 month follow up. The $p$ value for the association between the two parameters was 0.02 .

\section{Discussion}

In this study, we investigated the change in retinal thickness after laser photocoagulation treatment of diabetic macular oedema and its relation to visual acuity. We found that thicken- 


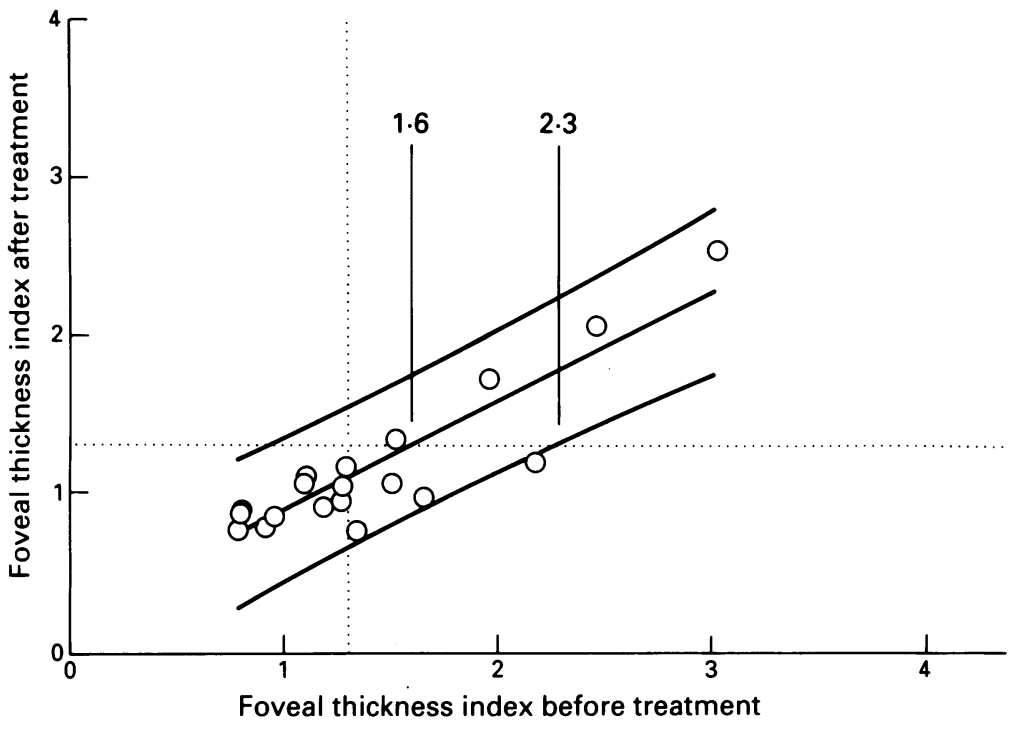

Figure 2 Relation between foveal thickening before and 4 months after treatment. The horizontal and vertical broken lines represent the upper limit of the $95 \%$ confidence interval for normal thickness; the solid line was derived by linear regression of the data points $(\mathrm{r}=0.89, p<0.01)$; limits of the $95 \%$ prediction confidence interval for the thickness index after treatment are indicated by solid lines. Pretreatment thickness indices of approximately 1.6 had nearly $50 \%$ probability for reversing the thickening to normal (thickness index $\leqslant 1 \cdot 3$ ) following treatment. ing decreased in $50 \%$ and remained unchanged in $36 \%$ of thickened locations 4 months after treatment, and new locations of thickening developed in only $3 \%$ of the locations. Thickening was completely resolved in $50 \%$ of locations with an average thickness index of $1 \cdot 6$. These results demonstrate that focal laser treatment seems to be effective in preventing the progression of macular oedema by reducing or maintaining the degree of thickening. In order unequivocally to relate thickness changes to laser treatment in patients with clinically significant macular oedema we had to compare them with a controlled group of patients in whom treatment was withheld, which of course would not be an ethical study. At the fovea, the high correlation between foveal thickness before and after treatment $(r=0.89, \mathrm{p}<0.01)$ indicated a strong dependence of the success of therapy, at 4 months, on the pretreatment thickening. In fact, from the linear regression parameters and the $95 \%$ prediction interval, we found that thickening of approximately $60 \%$ above the average normal value at the time of treatment had approximately a $50 \%$ probability for reversal of thickening to normal, while thickening greater than $130 \%$ above normal had a less than $2.5 \%$ probability. An improvement in visual acuity

Table 1 Foveal thickness and visual acuity before and after treatment

\begin{tabular}{|c|c|c|c|c|}
\hline \multirow[b]{2}{*}{ Case } & \multicolumn{2}{|c|}{ Foveal thickness index } & \multicolumn{2}{|c|}{ Visual acuity } \\
\hline & Before & After & Before & After \\
\hline 1 & $3 \cdot 0$ & $2 \cdot 6$ & $20 / 50$ & $20 / 40$ \\
\hline 2 & $2 \cdot 5$ & $2 \cdot 1$ & $20 / 50$ & $20 / 63$ \\
\hline 3 & $2 \cdot 0$ & 1.7 & $20 / 50$ & $20 / 63$ \\
\hline 4 & 1.5 & 1.4 & $20 / 20$ & $20 / 20$ \\
\hline 5 & $2 \cdot 2$ & $1 \cdot 2$ & $20 / 50$ & $20 / 40$ \\
\hline 6 & 1.5 & $1 \cdot 1$ & $20 / 32$ & $20 / 25$ \\
\hline 7 & 1.7 & 1.0 & $20 / 80$ & $20 / 40$ \\
\hline 8 & $1 \cdot 3$ & 0.8 & $20 / 50$ & $20 / 50$ \\
\hline 9 & $1 \cdot 3$ & $1 \cdot 2$ & $20 / 32$ & $20 / 20$ \\
\hline 10 & $1 \cdot 3$ & $1 \cdot 1$ & $20 / 16$ & $20 / 16$ \\
\hline 11 & $1 \cdot 3$ & 1.0 & $20 / 16$ & $20 / 20$ \\
\hline 12 & 1.2 & 0.9 & $20 / 20$ & $20 / 20$ \\
\hline 13 & $1 \cdot 1$ & $1 \cdot 1$ & $20 / 16$ & $20 / 20$ \\
\hline 14 & $1 \cdot 1$ & $1 \cdot 1$ & $20 / 32$ & $20 / 25$ \\
\hline 15 & $1 \cdot 1$ & $1 \cdot 1$ & $20 / 25$ & $20 / 20$ \\
\hline 16 & 1.0 & 0.9 & $20 / 25$ & $20 / 16$ \\
\hline 17 & 0.9 & 0.8 & $20 / 20$ & $20 / 20$ \\
\hline 18 & 0.8 & 0.9 & $20 / 20$ & $20 / 25$ \\
\hline 19 & 0.8 & 0.9 & $20 / 80$ & $20 / 125$ \\
\hline 20 & 0.8 & 0.8 & $20 / 12$ & $20 / 12$ \\
\hline
\end{tabular}

was observed at 4 months' follow up in $67 \%$ of the eyes with pretreatment visual acuities of 20/ 25 or worse. This finding is in agreement with previous studies that report stabilisation or improvement in visual acuity after treatment. ${ }^{4616}$ We have provided quantitative information indicating that foveal thickness within the normal range at follow up corresponded with improvement in vision, in seven out of eight eyes. The $p$ value for the association between pretreatment foveal thickening and visual acuity outcome was $0 \cdot 02$.

The quantitative evaluation of thickness change after treatment, although based on a single treatment at 4 months' follow up, confirms the qualitative findings of the ETDRS on the effectiveness of focal laser treatment of clinically significant macular oedema. ${ }^{9}$ Additionally, the quantitative nature of the method allowed objective evaluation of the degree of thickening and its relation with the treatment outcome. Additional studies with longer follow up periods and repeated treatment are necessary. In particular, there is a need to assess whether loss of vision attributed to oedema (and not to other factors such as ischaemia) will improve by reversing the thickening to normal. If so, monitoring of quantitative thickness in patients with diabetic macular oedema would provide a quantitative measure of the success of therapy and may help to identify a level of thickening optimally amenable to treatment, beyond which the odds of visual improvement are markedly reduced.

Presented in part at the Annual Meeting of Association for Research in Vision and Ophthalmology, Sarasota, FL, 28 April8 May 1991.

Dr Zeimer is entitled to sales royalty from Talia Technology, Inc, which is developing products related to the research described in this paper. In addition, the author serves as a consultant to Talia Technology, Inc. The terms of this arrangement have been reviewed and approved by Johns Hopkins University in accordance with its conflict of interest policies.

This study was supported in part by research grants from the Juvenile Diabetes Foundation International (New York, NY) (MS); Whitaker Foundation, Mechanicsburg, PA (RZ); and American Diabetes Foundation (Alexandria, VA) (RZ); by grant EY 6972 (RZ) and core grant EY 1792 (UIC Eye Center) from the National Eye Institute, Bethesda, MD; by a University of Illinois Scholar Award (RZ).

1 Patz A, Schatz H, Berkow JW, Gittelsohn AM, Ticho U. Macular edema - an overlooked complication of diabetic retinopathy. Trans Am Acad Ophthalmol Otolaryngol 1973; 77: $34-42$.

2 Ferris FLIII, Partz A. Macular edema. A complication of diabetic retinopathy. Surv Ophthalmol 1984; 28: 452-61.

3 Haik GM Jr, Terrell WLIII, Haik GM Sr. Diabetic retinopathy: a leading cause of new blindness. South Med F 1989; 82: 575-9.

4 Behrendt $T$. Therapeutic vascular occlusions in diabetic retinopathy. Argon laser photocoagulation. Arch Ophthalmol

5 Marcus DF, Aaberg TM. Argon laser photocoagulation treatment in diabetic cystoid maculopathy. Ann Ophthalmol 1977; 9: 365-72.

6 Wiznia RA. Photocoagulation of nonproliferative exudative diabetic retinopathy. Am $\mathcal{F}$ Ophthalmol 1979; 88: 22-7.

7 Olk RJ. Modified grid argon (blue-green) laser photocoagulation for diffuse diabetic macular edema. Ophthalmology 1986; 93: 938-50.

8 Otake H, Tanaka T, Ogura Y, Moritera T, Chihara E, Honda $\mathrm{Y}$. Macular photocoagulation for clinically significant macular edema. Fpn f Clin Ophthalmol 1990; 44: 951-4.

macular edema. Fpn f Clin Ophthalmol 1990; 44: $951-4$.
ETDRS Research Group. Photocoagulation for diabetic macular edema. Arch Ophthalmol 1985; 103: 1796-806.

10 Early Treatment Diabetic Retinopathy Study Research Group. Fundus photographic risk factors for progression of diabetic retinopathy. ETD

11 Early Treatment Diabetic Retinopathy Study Research Group. Classification of diabetic retinopathy from fluorescein angiograms: ETDRS Report Number 11. Ophthalmology 1991; 98: 807-22.

12 Shahidi M, Ogura Y, Blair NP, Rusin MM, Zeimer R. Retinal thickness analysis for quantitative assessment of diabetic macular edema. Arch Ophthalmol 1991; 109: 1115-7. 
13 Zeimer RC, Shahidi M, Mori MT, Benhamou E. In vivo evaluation of a noninvasive method to measure the retinal thickness in primates. Arch Ophthalmol 1989; 107: 1006-9.

14 Shahidi M, Zeimer RC, Mori M. Topography of the retinal thickness in normal subjects. Ophthalmology 1990; 97: $1120-4$.
15 Kleinbaum DG, Kupper LL. Applied regression analysis and other multivariable methods. North Scituate, Massachusetts: Duxbury Press, 1978: 37-70.

16 Lee CM, Olk RJ. Modified grid laser photocoagulation for diffuse diabetic macular edema. Long-term visual results. Ophthalmology 1991; 98: 1594-602.

\section{History of ophthalmology}

\section{Strange physical treatments}

Although surgery and therapeutics were practised to the limits of the period, the turn of the century ophthalmologist also relied heavily on a battery of physical treatments which have now disappeared.

Perhaps the least alarming of these was irrigation, albeit with esoteric substances - for example, extract of sheep adrenal, yellow psyocyanin, and methyl violet (which by irretrievably staining bed linen must have caused many instances of doctor-nurse enmity.

Regarding invasive therapy, injection into the loose subconjunctival tissue was practised in America and Europe with varying enthusiasm. Subconjunctival salt was supposed to quicken the lymphatic circulation, yet the procedure was extremely painful, even when cocaine was added, and patient compliance may have been a reason for its abandonment.

Ocular massage, first proposed by Donders, was practised widely to aid blood and lymph flow. Mercifully, simple digital pressure was often held to be sufficient. But for resistant cases 'massage traumatique' involved everting the cocainised lid and massaging vigorously with a finger dipped in boracic acid, until free bleeding was seen. Mechanical destruction of tissue occurred and indeed was intended, on the same principle as the curetting of scar tissue. One author adds that . . . 'cold compresses may be applied if the patient be unduly sore afterwards'. Instrumental massage of the globe was also performed with a flat, spoon-like instrument and was supposed to ripen cataract. Taking this principle further, Malakow produced a device terminating in a small ivory ball which vibrated at 9000 times a minute and could be applied to the diseased globe.

Although this was marketed enthusiastically, many practitioners stuck with simple jets of water, as 'pressure is produced by the weight of the solution falling onto the eye'. Steam massage was effected by applying a paper funnel to the nozzle of the ward tea kettle. The patient was coaxed, with lids held open, to within about two feet of the device and then progressively moved nearer. It is noted that astringents could be added to the kettle to medicate the steam, but presumably not while tea making was in progress! Heat was believed to cure deep inflammation, and poultices were very popular with the patients, although a simpler alternative was to direct steam jets onto dressings placed on the eye. For superficial inflammation 'cold' was prescribed, either in the form of a fish bladder filled with ice, or a Leiter coil made of metal tubing fed by a vessel filled with ice water.

For diplopia and neuralgia, electric current was thought to be beneficial. This was achieved by putting an electrode plate at the back of the patient's neck and a small anode on the closed lid. A current of about 3 milliamps was aimed for, and patients sometimes got 'unpleasant shocks' when the device was activated. Benedikt heroically reports using his own hand as electrode, to protect his patients from such surprises.

For ophthalmology, as for everything else, leeching was still advocated. Learning from recent advances in antisepsis, it is noted that only fresh, clean, and healthy leeches should be used. The latter was denoted by energetic contraction when grasped and a tendency to swim restlessly. Sluggish and apathetic leeches were not to be applied to patients as their drawing power was poor. Placing a rubber sheet with a small hole cut out over the patient's face spared him the sensation of the leech's wet body wriggling and of the trickle of blood. If the patient did not appear appetising, the leech could be tempted by a drop of sugared water, or of blood from the nurse's finger, applied to the temple.

It appears that the lot of the eye patient in the 1900 s consisted of (clinically sanctified) humane remedies such as hot baths, Turkish baths, rest, sleep, aromatic foot baths; and a nutritious diet was also recommended.

FIONA ROMAN

Fridenberg P. The ophthalmic patient. London: Macmillan, 1900. Juler HE. Ophthalmic science and practice. London: Smith, Elder and Co, 1983: 119-35. 